\title{
Über den Einfluss der Reizstärke auf die Tetanuskurve des Froschsartorius.
}

Von

Dr. Adolf Basler (Tübingen).

(Mit 7 Textfiguren.)

Nachdem sich in früheren Arbeiten eine Verschiedenheit der Zuckungskurve bei starken und schwachen Reizen in bezug auf die Dauer des Anstiegs herausgestellt hatte, lag es nahe, auch die Abhängigkeit des Tetanusverlaufs von diesen beiden. Faktoren zu prüfen.

Ich untersuchte deshalb auf Veranlassung von Herrn Professor v. Grützner und zum Teil in Gemeinschaft mit diesem das verschiedene Verhalten des Tetanus gegenüber schwachen und stärkeren Reizen. Es liegen zwar schon ausserordentlich sorgfältige und eingehende Untersuchungen über dieses Thema vor, so von Wedensky ${ }^{1}$ ) und $\mathrm{Hofmann}{ }^{2}$ ); doch ich wollte meine Versuche bei nicht so hohen Reizfrequenzen anstellen, um mich nicht $\mathrm{zu}$ sehr von dem Reizintervall $\mathrm{zu}$ entfernen, welches in der Natur vorkommt. Denn nach v. Kries ${ }^{3}$ ) beträgt die Zahl der Impulse beim Menschen auch für schnellste Bewegungen nur 15 in der Sekunde, beim Froscb, wo die Oszillationen der negativen Schwankung mit dem Kapillarelektrometer bestimmt wurden, nie über neun in der Sekunde.

Alle meine Versuche wurden im Sommer 1904 im Tübinger physiologischen Institut angestellt.

1) Wedensky, zit. bei Hofmann. Dieses Arch. Bd. 93 S. 186. 1902.

2) F. B. Hofmann, Über den Einfluss der Reizstärke auf den Tetanusverlauf. Dieses Arch. Bd. 95 S. 484. 1903.

3) J. v. Kries, Zur Kenntnis der willkürlichen Muskeltätigkeit. Arch. f. [Anat. u.] Physiol. Suppl. 1886 S. 1 (10). - Derselb e, Über die Abhängigkeit der Erregungsvorgänge von dem zeitlichen Verlauf der Elektrizitätsbewegungen. Arch. f. [Anat. u.] Physiol. 1884 S. 337 (371). 
Üb. den Einfl. der Reizstärke auf die Tetanuskurve des Froschsartorius. 345

\section{Methodik.}

Als Versuchsobjekt diente der Sartorius von Rana temporaria, der mit seinem Nerven auf die von mir (in diesem Arehiv Band 102 Seite 256) beschriebene Weise präpariert wurde. Dabei nahm ich nur insofern eine Änderung vor, als ich gleich bei Beginn der Präparation unter der Insertionssehne am Knie einen Faden durchzog und knotete, um eine bequeme Handhabe für den Muskel zu bekommen. Ausserdem hält auch die Sehne mit einer Fadenschlinge besser in der Klemme des Myographions.

Dieses Verfahren scheint mir vor dem von $K \ddot{u h n e}{ }^{1}$ ) angegebenen den Vorzug zu haben, dass sich das Präparat raseher herstellen lässt, und dass man den Nerven mit dem Plexus ischiadicus im Zusammenhang erhalten kann, während $K \ddot{u} h n$ e nur bis zum Austritt des Nerv. profund. post. aus dem Ischiadicus in die Höhe drang.

Es empfiehlt sich, den Nerven während der Präparation von Zeit zu Zeit mit physiologischer Kochsalzlösung anzufeuchten, da derselbe sehr dünn und deshalb leicht der Vertrocknung ausgesetzt ist. Sein Durchmesser beträgt an seinem Ende häufig nicht mehr als $0,1 \mathrm{~mm}$, wie ich mich mehrfach durch Messung mit dem Okularmikrometer überzeugen konnte.

Warum ich gerade den Sartorius verwendete, soll am Schluss der Arbeit erörtert werden.

Gereizt wurde mit Platinelektroden, und zwar für gewöhnlich der Plexus ischiadicus; doch blieb der Erfolg der gleiche, auch wenn ich den Nerven weiter abwärts erregte.

$\mathrm{Zu}$ sämtlichen Versuchen wurde das Grützner'sche Myographion ${ }^{2}$ ) mit einer Feder von $3 \mathrm{~g}$ Spannung benutzt. Da der Sartorius sich im Tetanus bedeutend verkürzt, wählte ich die geringste mögliche Vergrösserung $7: 2$.

Die Trommelgeschwindigkeit ist für jede Kurve aus den Zeitmarken zu ersehen, welche überall Sekunden angeben und mit dem graphischen Chronometer von Jaquet am Ende eines jeden Versuchs bei unverändertem Trommelgang aufgeschrieben wurden. Zur Reizung diente ein Bernstein'scher Induktionsapparat, teils mit,

1) W. Kühn e, Uber Muskelzuckungen ohne Beteiligung der Nerven. Arch. f. Anat. u. Physiol. 1859 S. 314 (317).

2) P. Grützner, Ein neues Myographion. Dieses Arch. Bd.31 S. 281. 1887. 
teils ohne Drahtbündel, durch dessen primäre Spule der Strom eines Leclanché-Elements ging, welcher von einem Wagner'schen Hammer unterbrochen wurde. Der Rollenabstand war so gross, dass die Sehliessungsinduktionsströme unwirksam waren. Der Öffnungsinduktionsstrom durchsetzte den Nerven stets in absteigender Richtung.

Der Wagner'sche Hammer selbst wurde von einem zweiten Stromkreis in Bewegung gesetzt, welcher ausserdem noch durch ein elektrisches Signal ging, das die Unterbrechungsfrequenz auf der Trommel unter jeder Kurve aufzeichnete.

Submaximaler und maximaler Tetanus bei verschiedenen Reizfrequenzen zwischen 20 und 35 . Schlägen in der Sekunde.

Nachdem der Sartorius im Myograpbion eingehängt war, stellte ich den Wagner'schen Hammer zunächst auf die gewünschte Schwingungszahl ein und entfernte die sekundäre Spule so weit von der primären, dass nach Unterbrechung des Nebenschlusses des sekundären Stromkreises (durch Herausziehen des Stöpsels) sich keine Reizwirkung am Muskel zeigte. Nun suchte ich durch langsames Annähern der Spulen die Reizstärke, bei der sich der. Muskel eben zu kontrahieren begann. Dies war in der Regel der Fall bei einem Rollenabstand von ungefähr $35 \mathrm{~cm}$. Hierauf wurde abgeblendet und, nachdem die Trommel in Gang gesetzt war, mit dieser Stromstärke gereizt. Jetzt geriet der Muskel in Tetanus, wobei er sich aber nur um einen Bruchteil der ganzen möglichen Verkürzung zusammenzog. Näherte man nun die beiden Spulen, dann trat eine Stellung derselben ein, bei welcher der Sartorius in maximalen Tetanus geriet. Dies geschah gewöhnlich bei einem Rollenabstand von ungefähr $30 \mathrm{~cm}$.

Es sei noch bemerkt, dass in einigen Fällen auf Reizung mit schwachen Strömen im Anfang nur eine Folge von unregelmässigen Zusammenziehungen auftrat; sobald aber der Muskel nur sehr kurze Zeit tetanisiert war, blieb der Hebel auch bei schwachen Reizen oben, so dass man auch in diesen Fällen mit Recht von einem submaximalen Tetanus sprechen kann.

Umstehende Kurve dürfte die Verhältnisse am besten zeigen.

Berücksichtigt man die von Grützner ${ }^{1}$ ) seit lange vertretene Ansicht, dass die beiden im Froschmuskel vorkommenden Faserarten

1) P. Grützner, Zur Anatomie und Physiologie der quergestreiften Muskeln. Recueil zoologique Suisse 1884 p. 665. 
eine verschiedene physiologische Bedeutung haben, bcsonders die Ergebnisse meiner früheren Untersuchungen (dieses Arch. Bd. 102 S. 254. 1904), wonach die in geringer Stärke vorhandenen protoplasmareichen dünnen Fasern leichter erregbar sind als die an Kraft äberlegenen protoplasmaarmen dicken, so ist es ziemlich sicher, dass auch beim Tetanus ein ähnliches Verhalten vorliegt, indem bei schwachen tetanisierenden Reizen die dunnen Fasern in Aktion treten, bei starken Reizen dagegen auch noch die dicken. Da aber die dünnen Fasern in ihrer Gesammtheit schwächer sind als die dicken, vermögen sie die an dem Mukkel ziehende Last nicht so

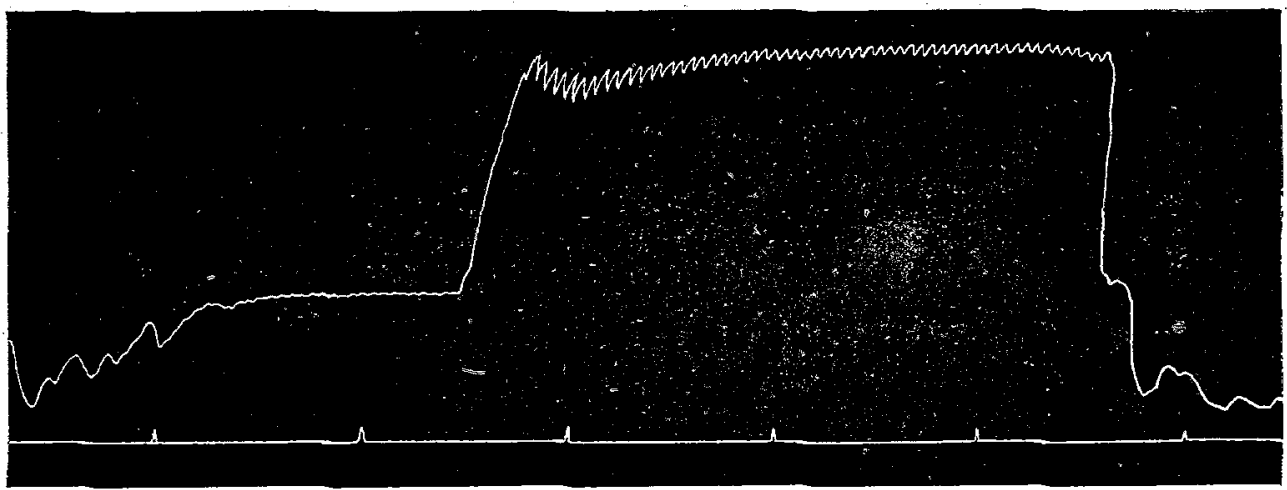

Fig. 1. Tetanuskurve des Sartorius. Versuch vom 25. Mai 1904. Zimmertemp. $22^{\circ} \mathrm{C}$. Spannung $3 \mathrm{~g}$. Zeitmarken $=$ Sek. Reizfrequenz beim submaximalen und beim maximalen Tetanus ung. 20 Schläge in der Sek. Wo die Kurve plötzlich steigt, wurden die Rollen genähert.

hoch zu heben wie die an Kraft überlegenen dicken, welche erst einsetzen, wenn der Reiz eine gewisse Stärke überschritten hat.

Dass diese Annahmen zutreffend sind, scheint mir aus folgenden, schon anderweitig erwähnten Tatsachen hervorzugehen, die ich wegen ihrer grundsătzlichen Wiebtigkeit hier noch einmal kurz zusammenstellen möchte.

Wird der Ischiadicus des enthäuteten Froschschenkels mit schwachen tetanisierenden Induktionsströmen gereizt, so tritt Beugung ein, bei Verstärkung der Ströme dagegen Streckung (RitterRollett'sches Phänomen). Die Beuger, die bedeutend mehr dünne Fasern enthalten als die Strecker (Grutzner) ${ }^{1}$ ), werden also durch geringere Reize in Tetants versetzt als die Extensoren.

1) K. Bonhöffer, Über einige physiologische Eigenschaften dünn- und dickfaseriger Muskeln usw. Dieses Arch. Bd. 47 S. 125.1889. 
Der von seinem Nerven aus tetanisierte Sartorius des Frosches krümmt sich bei Reizung mit schwachen Strömen nach vorn; bei Reizung mit starken zieht er sich im ganzen zusammen ${ }^{1}$ ). An der vorderen Seite dieses Muskels liegen aber überwiegend dünne Fasern (Grützner) ${ }^{2}$ ). Aus diesen beiden Versuchen geht hervor, dass die dünnen Fasern leichter erregbar sind als die dicken. Ähnlich verhält sich der Gastrocnemius und wahrscheinlich mehr oder weniger jeder Froschmuskel. Bringt man des weiteren einen Muskel (am besten den Sartorius) durch schwache Reizung von seinem Nerven aus zu einer niederen Zuckung, dann ist seine Anstiegszeit grösser als für einen starken Reiz, was also beweist, dass die dünnen, leichter erregbaren Fasern langsamer zucken als die schwer erregbaren dicken. Hiernach muss nach allem, was wir über die Entstehung des Tetanus wissen, für schwache Reize eine geringere Frequenz genügen, um einen glatten Tetanus zu erhalten, als für starke. Oder eine bestimmte Reizfrequenz nuss beï submaximaler Reizung einen tonischen, bei maximaler einen klonischen Tetanus erzeugen.

Die Richtigkeit dieser Überlegung wurde durch den. Versuch erwiesen, indem leicht eine bestimmte Reizfrequenz zu finden ist, bei welcher der submaximale Tetanus glatt, der maximale dagegen klonisch ist.

Auf Grund mehrerer solcher Versuche gelangte ich zu folgenden Ergebnissen:

Bei einer Frequenz von etwa zwanzig Schlägen in der Sekunde tritt ein klonischer Tetanus ein sowohl bei maximaler wie bei submaximaler Reizung; doch sind die Zacken höher bei der letzteren. Steigt die Zahl der Impulse, so wird der submaximale Tetanus immer glatter, bis er bei ungefähr 30 Stössen vollständig tonisch ist, während bei dieser Reizfrequenz der maximale noch deutliche Zacken erkennen lässt. Steigt die Zahl der Reize noeh mehr, dann wird bei ungefähr 34 Schlägen in der Sekunde auch das hohe Plateau der Kurve glatt.

Als Beleg seien zwei Versuche angeführt.

1) A. Basler, C̈ber den Einfluss der Reizstärke und der Belastung auf die Muskelkurve. Dieses Arch. Bd. 102 S. 254 (266). 1904.

2) G: Zenneck, Über die chemische Reizung nervenhaltiger usw. Skelettmuskeln. Dieses Arch. Bd. 76 S. 21 u. 56. 1899. 
Üb. den Einfl. der Reizstärke auf die Tetanuskurve des Froschsartorius. 349

\section{Versuch vom 13. Juni 1904.}

Zimmertemp. $24^{\circ}$ C. Mittelgrosse männliche Rana temp. Dauer des Versuchs $10^{\mathrm{h}}$ bis $10^{\mathrm{h}} 45^{\prime}$. Reizung des Sartorius vom Plexus ischiad, aus.

\begin{tabular}{|c|c|c|}
\hline $\begin{array}{l}\text { Zahl der } \\
\text { Reize in der } \\
\text { Sekunde }\end{array}$ & $\begin{array}{c}\text { Submaximaler } \\
\text { Tetantıs }\end{array}$ & Maximaler Tetanus \\
\hline $\begin{array}{l}23 \\
27 \\
30 \\
34\end{array}$ & $\begin{array}{l}\text { kleine Zacken } \\
\text { glatt } \\
\text { glatt } \\
\text { glatt }\end{array}$ & $\begin{array}{l}\text { grosse Zacken } \\
\text { mittlere Zacken } \\
\text { kleine Zacken } \\
\text { glatt }\end{array}$ \\
\hline
\end{tabular}

Fersuch rom 14. Juni 1904.

Zimmertemp. $24,5^{\circ}$ C. Grosse männliche Rana temp. Dauer des Versuchs $9 \mathrm{~h}$ bis $9 \mathrm{~h} 50$ \% Reizung des Sartorius von der Mitte des Nerven aus.

\begin{tabular}{|c|c|c|}
\hline $\begin{array}{l}\text { Zahl der } \\
\text { Reize in der } \\
\text { Sekunde }\end{array}$ & $\begin{array}{c}\text { Submaximaler } \\
\text { Tetanus }\end{array}$ & Maximaler Tetanus \\
\hline $\begin{array}{l}24 \\
26 \\
32 \\
35 \\
44\end{array}$ & $\begin{array}{l}\text { kleine Zacken } \\
\text { sehr kleine Zacken } \\
\text { glatt } \\
\text { glatt } \\
\text { glatt }\end{array}$ & $\begin{array}{l}\text { grosse Zacken } \\
\text { mittlere Zacken } \\
\text { sehr kleine Zacken } \\
\text { glatt } \\
\text { glatt }\end{array}$ \\
\hline
\end{tabular}

Für die drei Typen des Tetanus bei verschiedener Reizfrequenz mögen folgende drei Kurven als Beispiel dienen.

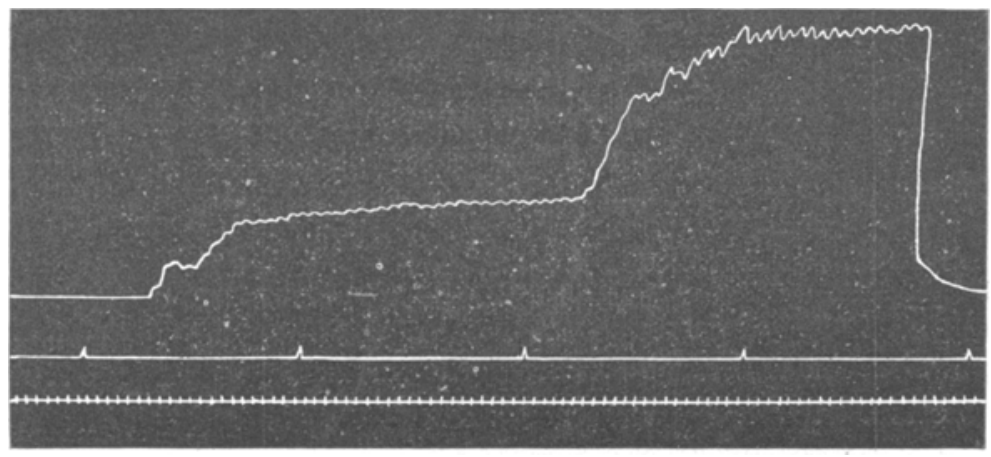

Fig. 2. Tetanuskurve des Sartorius. Versuch vom 23. Juni 1904. Zimmertemp. $24,5^{\circ}$ C. Spannung 3 g. Zeitmarken (obere Linie) = Sek. Reizfrequenz (untere Linie) beim submaximalen und beim maximalen Tetanus ungefähr 22 . 


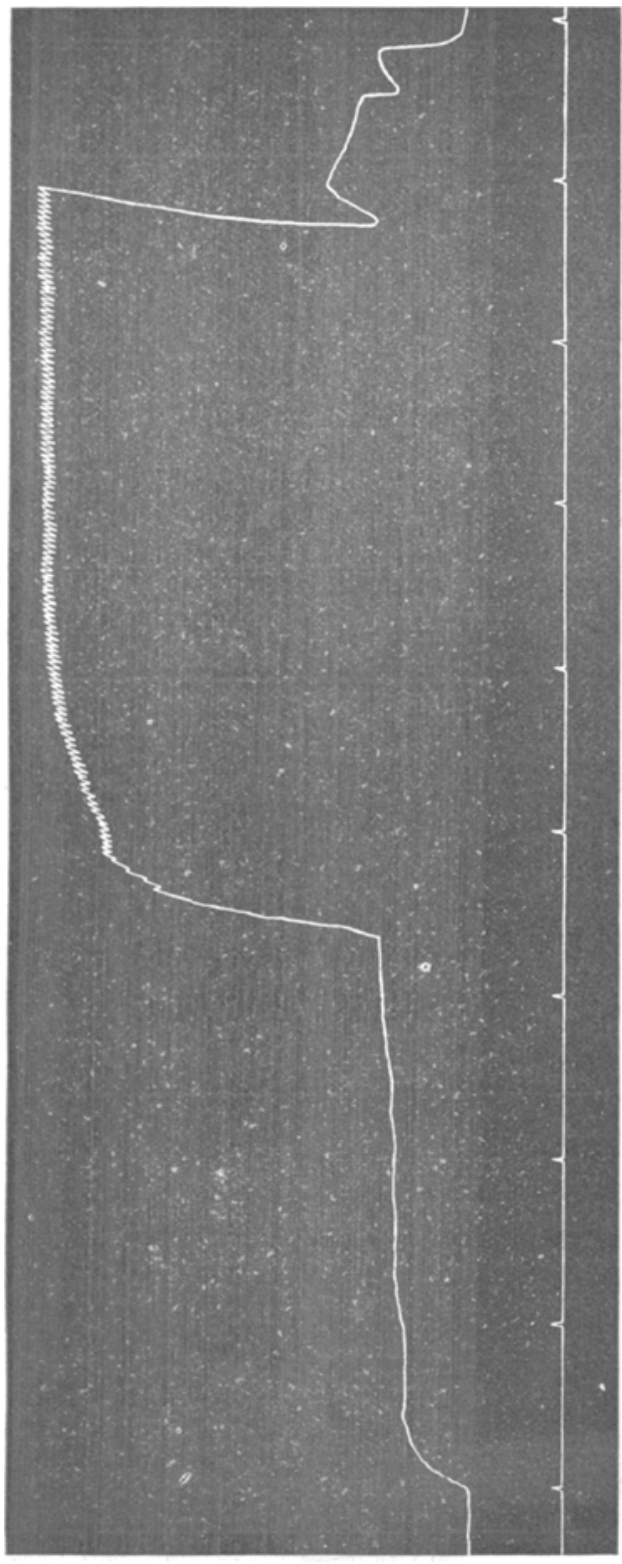

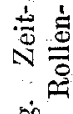

$\infty \frac{1}{8}$

$\stackrel{80}{3}$

:

की

ن

- 8

跍

28

크

赵

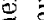

通 S $E$

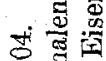

实

焉造

․ㅗ

s

톨

영

贾

$5 \approx$

证

$\Xi$

递

os

惌

$\$$

畓

胥

忢

$\leftrightarrow$ II

क

为苟 


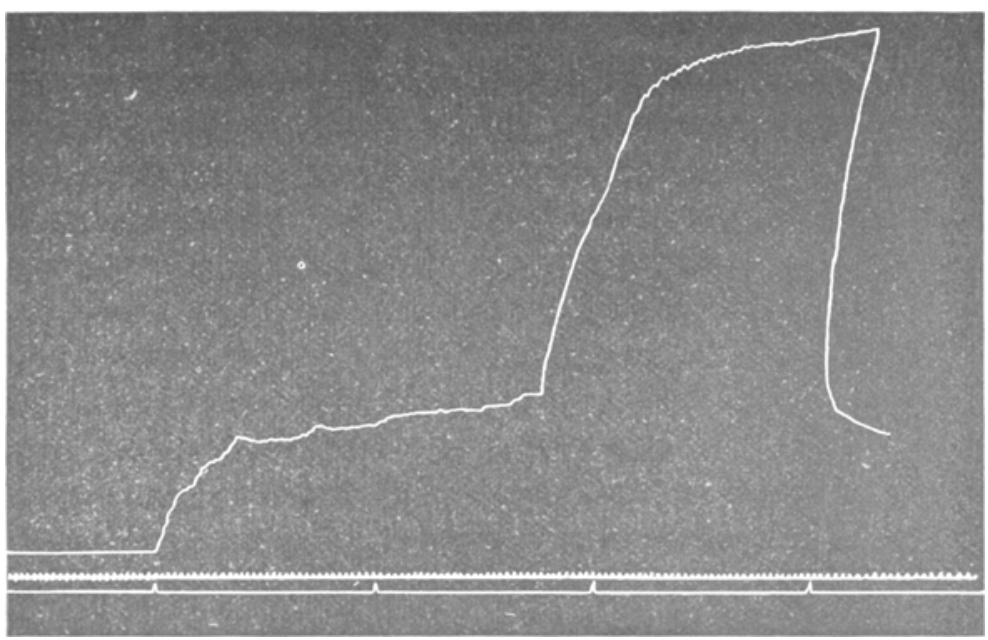

Fig. 4. Tetanuskurve des Sartorius. Versuch vom 24. Juni 1904. Zimmertemp. $24,5^{\circ}$ C.: Spannung 3 g. Zeitmarken (untere Linie) $=$ Sek. Reizfrequez (obere Linie) ungefähr 34 Schläge in der Sek. 34 Schläge war die niederste Frequenz, bei der der maximale Tetanus nahezu glatt wurde. Deshalb sind im Anfang des maximalen Tetanus auch kleine Zacken vorhanden.

\section{Bemerkungen zu obigen Versuchen.}

Die angegebenen Zahlen sollen nur das Verhältnis der Reizfrequenzen illustrieren, nicht aber als absolute Grössen gelten, denn je nach dem Präparat (wahrscheinlich abhängig von seiner Empfindlichkeit) müssen die Reizstösse sich in verschiedener Geschwindigkeit folgen, damit man das gewünschte Aussehen des Tetanus erhält, doch entsprechen die angegebenen Zahlen dem gewöhnlichen Ergebnis meiner Untersuchungen.

Es sei noch erwähnt, dass die Zusammenstellung gemacht wurde auf Grund von Versuchen hei einer Zimmertemperatur von 24 bis $24,5^{\circ}$ C. Bei dem grossen Einfluss, den die Temperatur auf die Art der Muskelkontraktion ausübt (ich erinnere nur an die eingehenden Untersuchungen von $G$ ad und Heymanns) ${ }^{1}$ ), mag es damit zusammenhängen, dass bei der auf Fig. 1 dargestellten Kurve der submaximale Tetanus schon bei einer Reizfrequenz von 19-20 beinahe glatt ist. Dieser Versuch wurde nạmmlich ausnahmsweise bei einer Temperatur von $22^{\circ} \mathrm{C}$. angestellt.

1) J. Gad und J. F. Heymans, Über den Einfluss der Temperatur auf die Leistungsfähigkeit der Muskelsubstanz. Arch. f. [Anat. und] Physiol. 1890. Suppl. S. 59. 
Ein Fehler in dem Sinne, dass durch Annäherung der Rollen die Schliessungsströme wirksam werden, kommt für die beschriebenen Versuche nicht in Betracht, weil dadurch die Reizfrequenz vermehrt, also der Tetanus glatter werden müsste. Man kann sich auch durch Abzählen der Zacken leicht überzeugen, dass bei Verstärkung des Reizes die Frequenz die gleiche bleibt. Ausserdem stellte ich fest, dass bei dem zu meinen Versuchen nötigen Rollenabstand einzelne Schliessungsschläge noch lange keine Zuckungen hervorriefen.

Ebensowenig können die beschriebenen Erscheinungen durch Ermüdung vorgetäuscht werden, da auch diese die Tetanuskurve glatter werden liesse, - ein Einfluss, den ich auch bei meinen Untersuchungen häufig direkt beobachten konnte. So ist z. B. in Fig. 1 eine deutliche Abnahme der Zackenhöhe gegen das Ende des Tetanus hin zu erkennen.

Die Ergebnisse meiner Versuche, dass der Tetanus bei stärkeren Reizen böhere Zacken aufweist als bei schwachen, stehen nicht vereinzelt da; namentlich lassen sich gewisse Analogien aufstellen mit dem willkürlichen Tetanus des lebenden Menschen. So weist Brücke ${ }^{1}$ ) auf die Tatsache hin, dass unsere Faust zu zittern beginnt, sobald wir den Arm eine forcierte Beugung ausführen lassen.

Wenn Grützner ${ }^{2}$ ) und Hartmann ${ }^{3}$ ) vielfach ein von meinen Resultaten abweichendes Verhalten gefunden haben, indem durch Verstärkung des Reizes der Tetanus glatter wurde, so kann ich mir den Gegensatz nur dadurch erklären, dass die genannten Forscher stets mit dem Gastroenemius arbeiteten, welcher sich, wie später gezeigt werden soll, in seinem physiologischen Verhalten hierin wesentlich von dem Sartorius unterscheidet.

Es erhalten eben - worauf hier besonders hingewiesen sei - alle Untersuchungen über die Entstehung des Tetanus plötzlich ein ganz anderes Gesicht, wenn man sich in der Art der Erregung mehr an die natürlichen Verhältnisse anschliesst, und wenn man vor allem den Bau und die Zusammensetzung des Muskels aus zwei ganz verschiedenen Elementen berücksichtigt.

1) E. Brücke, Über willkürliche und krampfhafte Bewegungen. Sitzungsberichte der Wiener Akademie Bd. 76 Abteilung III S. 237 (239). 1877.

2) P. Grützner, Reizwirkungen der Stöhrer'schen Maschine. Dieses Arch. Bd. 41 S. 256.1887.

.) J. Hartmann, Zur Mechanik des quergestreiften Muskels. Dissertation S. 39. Tübingen 1903. 
Ub. den Einfl. der Reizstärke auf die Tetanuskurve des Froschsartorius. 353

Dass aber auch der Gastrocnemius unter gewissen, mir unbekannten Bedingungen ähnliche Ergebnisse liefern kann, geht aus den Untersuchungen von Kohnstamm ${ }^{1}$ ) hervor, der nachweisen konnte, dass die Zacken des Tetanus durch Verstärkung des Reizes höher werden.

\section{Langsame Bewegung, durch künstliche Reize hervorgebracht.}

Lässt man den submaximalen Tetanus sich zunächst nur wenig über die Abszissenachse erheben, was bei genügender Abschwächung des Reizes leicht gelingt, dann kann man auf diese Kurve durch geringe Annäherung der Spulen eine neue aufsetzen, welche noch lange nicht die maximale Höhe erreicht hat. Verstärkt man die Schläge noch mehr, so verkürzt sich der Muskel von neuem. Man bekommt auf diese Weise eine Tetanuskurve von der Gestalt einer Treppe; jedes Ansteigen entspricht einer Verstärkung des Reizes. Natürlich treten, wie nach den früheren Ausführungen zu erwarten war, bei jeder Erhebung höhere Zacken auf.

Als Beleg sei auf S. 354 eine Kurve dieser Art wiedergegeben.

Diese Erscheinung lässt sich wohl am besten, wie folgt, erklären: Solange der Reiz wenig wirksam ist, werden nur dünne Fasern erregt und von diesen sogar zunächst nur ein Teil. Die schwachen Fasern können den Muskel nicht hoch heben; deshalb bleibt der Tetanus nieder und bei mittlerer Frequenz glatt, denn es treten nur langsame Fasern in Aktion. Wird der Strom verstärkt, so nehmen mehr Fasern an der Arbeit teil, vielleicht alle dünnen. Der Tetanus wird höher, weil die sich kontrahierenden Elemente jet kräftiger sind, doch er bleibt glatt; oder aber es haben sich schon einige wenige dicke Fasern beteiligt, dann treten Zacken auf, indessen, da die erregten dicken Fasern nur in geringer Zahl und deshalb schwach sind, können sie sich auch nur wenig kontrahieren; die Zacken bleiben klein. Erst wenn durch weiteres Verstärken des Stroms viele dicke Fasern erregt werden, kann sich der Muskel mehr kontrahieren, und die Zacken werden höher.

Ein solches Verhalten des Muskels, wobei derselbe sich ruckweise bis zu einer gewissen Höhe verkürzt, unterscheidet sich also nur graduell von einer der natïrlichen ähnlichen Bewegung; indem

1) 0. Kohnstamm, Experimentelle Untersuchungen zur Analyse des Tetanus. Arch. f. [Anat. u.] Physiol. 1893 S. 125.

E. Pflüger, Archiv für Physiologie. Bd. 105. 


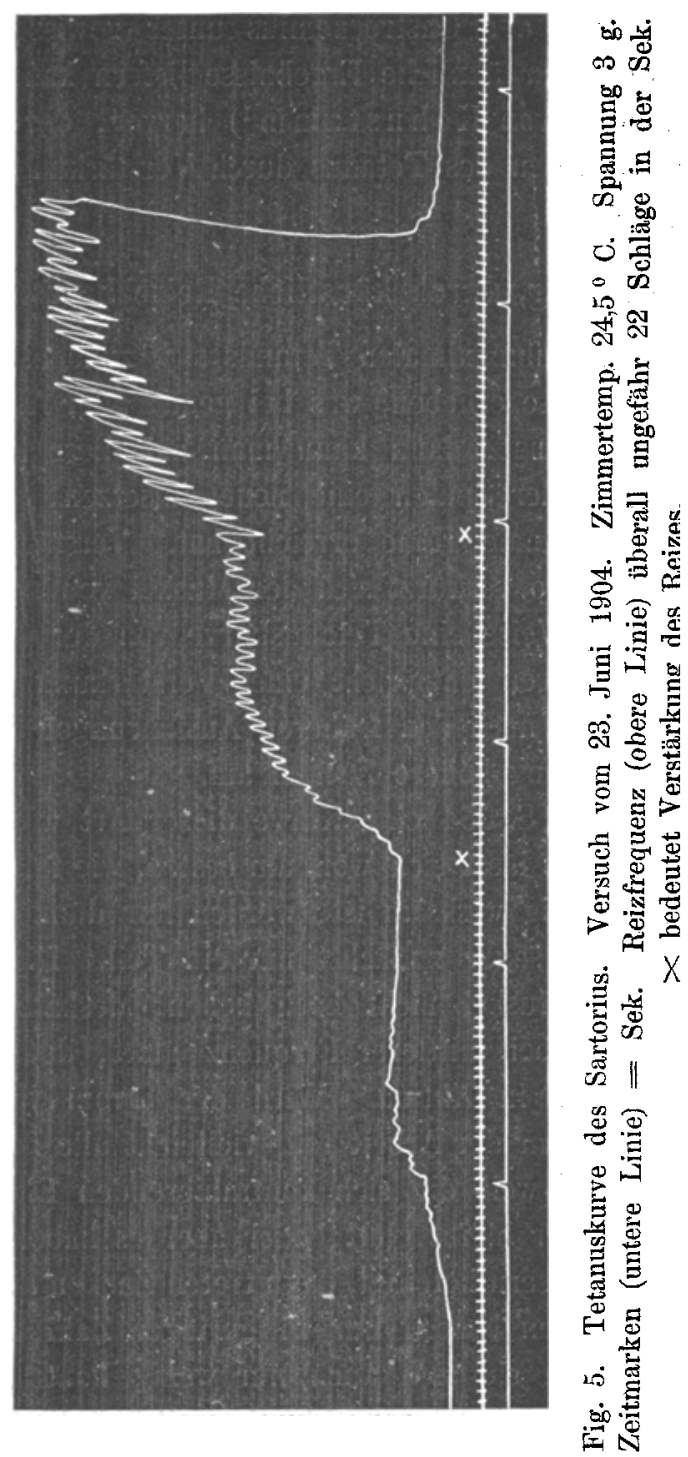

dort die Impulse nicht in Absätzen verstärkt werden, sondern so allmählich, dass statt der treppenformigen eine ganz langsame Verkürzung eintritt.

Mit dieser Auseinandersetzung habe ich im wesentlichen, wie schon mehrfach erwähnt, die von Grützner ${ }^{1}$ ) seit lange vertretene

1) P. v. Grützuer, Wanderversammlung der südwestdeutschen Neurologen und Irrenärzte. Baden-Baden 1904. 
Üb. den Einfl. der Reizstärke auf die Tetanuskurve des Froschsartorius. 355

Ansicht wiedergegeben, die er erst kürzlich wieder aussprach. Bei dieser Gelegenheit wies er auch auf die Bedeutung der Tatsache hin, dass man durch schwache Reize nur wenig, durch starke viele Muskelfasern in Tetanus versetzen kann, und dass, wenn man durch Verstärkung der Reize diesen Vorgang allmählich vor sich gehen lässt, Schritt für Schritt immer mehr Fasern in Tätigkeit geraten, wodurch eine ganz langsame, der natürlichen Bewegung unserer Muskeln ähnliche Zusammenziehung erhalten werden kann.

Zur Ausführung eines solchen Versuchs darf man nach Grützner nicht, wie es bisher fast immer geschehen ist, mit maximalen Reizen einfach auf alle Fasern gewissermassen "darauf loshauen", sondern man muss die Intensität der Reizimpulse möglichst langsam ansteigen lassen. Es empfiehlt sich daher, das Element so stark zu wählen, dass man die Spulen sehr weit auseinander entfernen kann, so dass eine verhältnismässig grosse Verschiebung derselben nur einen kleinen Unterschied der Reizstärke verursacht.

Auf diese Weise gelingt es bei vorsichtigem Annähern der sekundären Spule leicht, ein allmähliches Ansteigen des Tetanus zu erhalten, wie dies die auf S. 356 wiedergegebene Kurve beweist.

So konnte Grützner durch Reizung des Hüftnerven mit allmählich verstärkten tetanisierenden Induktionsströmen eine langsame - der natürlichen Bewegung ähnliche - Beugung des Froschschenkels und bei weiterer Verstärkung des Reizes eine langsame Streckung desselben dann sehr schön beobachten, wenn das Präparat sich während des Versuchs unter physiologischer Kochsalzlösung befand. Wurden die Ströme abgeschwächt, so ging die Streckung wieder langsam in Beuqung über. Zu diesem Experiment eignen sich nicht alle Frösche gleich gut. Am besten gelang dasselbe während des Winters. Die Versuchsanordnung war folgende: Die beiden enthäuteten hinteren Extremitäten eines Frosches wurden in vertikaler Richtung in ein mit physiologischer Kochsalzlösung gefülttes, zur bequemen Beobachtung vorn mit einer Glaswand versehenes Gefäss gebracht, gerade so tief, dass der Plexus ischiadicus beiderseits über die Flüssigkeit herausragte. An den einen, oben durchschnittenen Nerven war ein Faden gebunden, so dass derselbe leicht über ein zur Reizung dienendes Elektrodenpaar gelegt werden konnte.

Um einen Tetanus des Sartorius zu erzielen, der proportional der Zeit, also in einer schiefen Geraden ansteigt, muss die Intensität der Reizimpulse zuerst nur wenig, später immer mehr zunehmen. Aus 


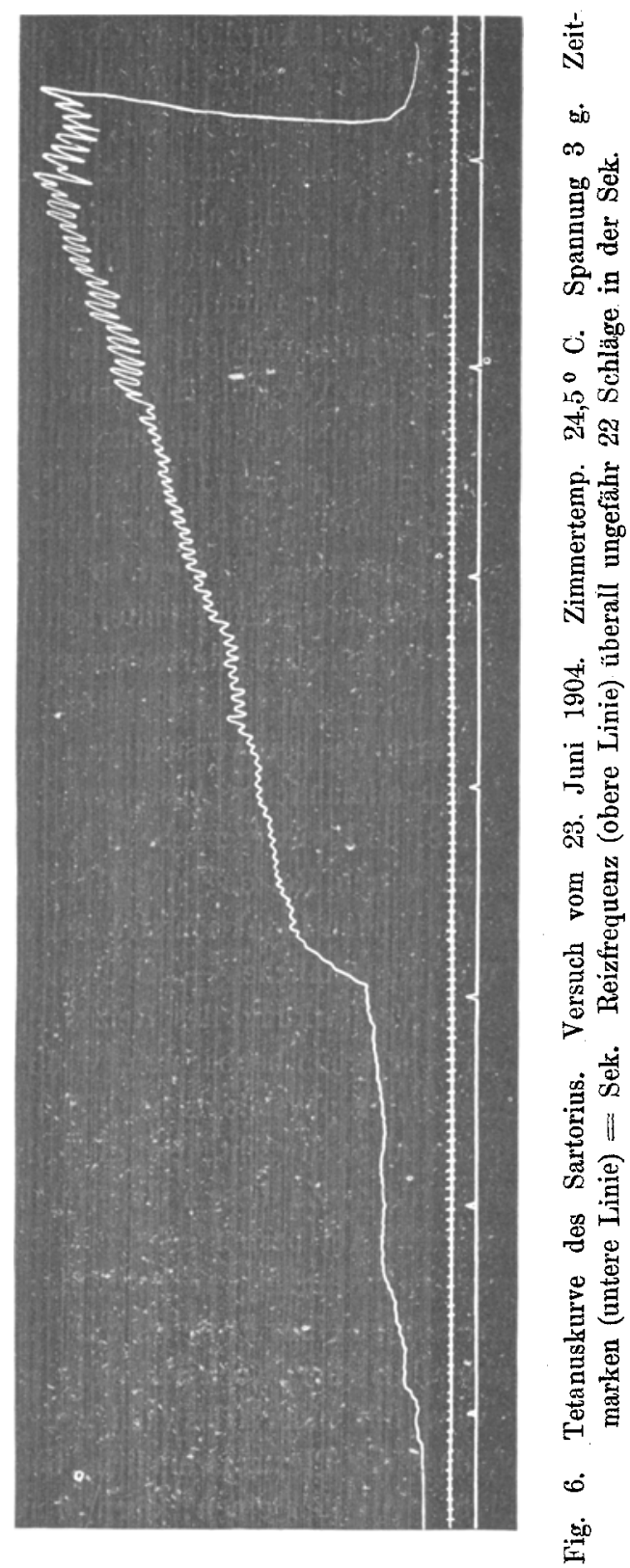

diesem Grunde ist auch gerade der erste Teil einer Kurve mit langsamem Anstieg am schwierigsten zu erhalten, weil dort sehr leicht die Verschiebung der sekundären Spule zu stark wird und deshalb eine vorübergehende plötzliche Verkürzung erfolgt. 
Üb. den Einfl. der Reizstärke auf die Tetandsklưve des Froschsartorius. 357

Wie verhält sich diese külstlich hervorgebrachte Kontraktionsform zur willkuirlichen Bewegung?

Nach Brück $\mathrm{e}^{\mathbf{1}}$ ) werden: die natürlichen Bewegungen dadurch reguliert, dass, wenn die zu überwindende Last zu klein wäre, um eine langsame Verkürzung des Muskels zu bedingen, dureh die Antagonisten künstliche Widerstände gesetzt werden. v. Kries ${ }^{2}$ ) gelangte $z u$ der Anschauung, dass auch kurze Bewegungen stets unter Mitwirkung von Antagonisten ausgeführt werden. So sicher diese Tatsache erwiesen ist, ebenso allgemein wird auch die Fähigkeit jedes Muskels, für sich allein sich beliebig langsam zu kontrahieren, angenommen.

Wie können wir uns nun vorstellen, dass in der Natur eine Bewegung mit beliebiger Langsamkeit ausgeführt wird?

Wie namentlich von Grützner vielfach betont wurde, beteiligen sich an einer langsamen Muskelbewegung zuerst nur wenig Fasern, die ein bestimmtes Gewicht nur bis zu einer geringen Höhe heben. Hierauf setzt eine andere geringe Anzahl von Fasern ein, welche die alten bei ihrer Arbeit unterstützen; so dass wieder ein kleines Übergewicht auf Seite des Muskels besteht. Sobald das Gewicht wieder mit der Kraft der Muskelfasern im Gleichgewicht ist, treten auch wieder neue Elemente hinzu usf.

Durch dieses allmähliche Eintreten einzelner Fasern bezw. Fasergruppen in die Aktion, sowie namentlich durch die Verwendung von langsamen (wesentlich roten) und schnellen (wesentlich weissen) Fasergruppen wird nach Grützner gerade für unsere langsamen Bewegungen (man denke nur an die Führung eines Pinsels oder einer Schreibfeder) jene Sicherheit verständlich und gewährleistet, die eben für feine Bewegungen der Hand notwendig ist.

Berücksichtigt man weiter die Grützner'sche Ansicht, dass bestimmte Nerven- und Muskelfasergruppen ein zusammengehöriges Ganzes bilden, so wird man hiernach annehmen müssen, dass in der Natur nicht durch den ganzen Nerven, d. h. durch alle seine Fasern, ein gleichzeitiger Impuls von bestimmter Stärke geht, sondern dass vom Zentralorgan aus nur diejenigen Nervenfasern in Erregung ver-

1) E. Brücke, Über willkürliche und krampfhafte Bewegungen. Sitzungsberichte der Wiener Akademie Bd. 76 Abteilung III S. 237 (259). 1877.

2) J. v. Kries, Zur Kenntnis der willkürlichen Muskeltätigkeit. Arch. f. [Anat. u.] Physiol. 1886 Suppl. S. 1 (10). 
setzt werden, deren Muskelfasern sich verkürzen sollen. Demnach würde eine langsame natürliche Bewegung dadurch zustande kommen, dass vom Zentralorgan aus zuerst nur eine geringe Zahl von Nervenfasern mit ihren Múskelelementen Reizimpulse erhält, die im Verlauf der Tätigkeit stetig vermebrt wird.

Während es im Jahre 1859 du Bois-Reymond ${ }^{1}$ ) gelang, am Muskel eines mit Strychnin vergifteten Froscbes die Aktionsströme durch eine Reihe von getrennten Zuckungen eines sekundären Nerv-Muskelpräparats nachzuweisen, konnte Harless ${ }^{2}$ ) im Jahre 1862 zeigen, dass eine natürliche Muskelbewegung im günstigsten Falle nur eine Zuckung im Beginne der Kontraktion auslöst, niemals aber einen sekundären Tetanus. Diese Tatsache führte $\mathrm{Brücke}{ }^{3}$ ) zu der Auffassung, dass bei einer natürlichen Bewegung eine Muskelfaser nach der anderen gleich dem Pelotonfeuer erregt wird. Hierdurch würden dann auch die elektrischen Vorgänge so auseinandergezogen, dass man, um bei dem Beispiel zu bleiben, anstatt einer krachenden Salve einen langgezogenen Donner hört. Dieser langgezogene elektrische Donner aber ist auf den secundären Nerven physiologisch unwirksam; nur die Salve ist wirksam.

Nach den Versuchen von v. $\mathrm{Kries}^{4}$ ), welche es sehr wahrscheinlich machen, dass die willkürlichen Impulse Zeitreize sind oder ihnen sehr nahestehen, ist jedoch diese Auffassung nicht mehr notwendig. Denn wie v. Kries gezeigt hat, werden bei langsam ansteigenden Reizen die elektrischen Vorgänge so gedehnt, dass sie unter Umständen auf ein sekundäres Froschpräparat vollständig unwirksam werden.

Lovén ${ }^{5}$ ) und v. Kries ${ }^{6}$ ) konnten nachweisen, dass die Fre-

1) E. du Bois-Reymond; Untersuchungen über tierische Elektrizität Bd. 2 S. 515. Berlin 1849.

2) E. Harless, Analyse der willkürlichen Bewegung. Zeitschr. f. rationelle Medicin III. Reihe Bd. 14 S. 97 (110). 1862.

3) E. Brücke, 1. c. S. 263.

4) J. v. Kries, Über die Abhängigkeit der Erregungsvorgänge von dem zeitlichen Verlauf der Elektrizitätsbewegungen. Arch. f. [Anat: u.] Physiol. 1884 S. 337 (369).

5) C. Lovén, Zur Frage von der Natur des Strychnintetanus und der willkürlichen Muskelkontraktion. Zentralbl. für die mediz. Wissensch. S. 113 (114). 1881.

6) J. v. Kries, I. c. 
Üb. den Einfl. der Reizstärke auf die Tetanuskurve des Froschsartorius. 359

quenz des willkürlichen Tetanus eine viel kleinere ist, als man bisher annahm, indem dieselbe beim Frosch neun Oszillatationen in der Secunde nicht überschreitet.

Wie verhält sich dazu die künstliche Reizung? Bei meinen Versuchen wurde der Nerv mit einer Folge von Induktionsströmen gereizt, die fuerst schwach waren, aber an Intensität immer mehr zunahmen. Nun machen es 'die neuesten Ergebnisse yon Goteh ${ }^{1}$ ) sehr wahrscheinlich, dass bei Reizung eines Nerven mit schwachen Strömen nur wenig Nervenfasern, diese wenigen aber maximal, bei starker Reizung dagegen alle getroffen werden oder wenigstens ein grosser Teil derselben. Jede in Erregung versetzte Nervenfaser veranlasst die zu ihr gehörige Muskelfaser zur Kontraktion.

Nach dieser von. Grützner seit lange vertretenen Ansicht würde es also keinen wesentlichen Unterschied bedingen, ob man den ganzen Nerven mit schwachen Strömen behandelt, wodurch eben immer nur einige Nervenfasern mit ihren Muskelfasern erregt werden, oder ob vom Zentralorgan aus wenig Nervenfasern in Aktion versetzt werden, wie anderseits ein starker Reiz einem in viele Nervenfasern geschickten Impulse entṣpräche.

Dagegen in der Art des Reizes unterscheidet sich diese künstlich herbeigeführte langsame Verkürzung. Denn als Reiz wurden die sehnell ansteigenden Öffnungsinduktionsströme verwendet, von denen, wie eben erwähnt, die natürlichen Impulse offenbar bedeutend abweichen. Um so interessanter scheint mir daher die Tatsache, dass es unter gewissen Bedingungen auch mit einer Folge schnell ansteigender Reize gelingt eine allmählich ansteigende Muskelverkürzung zu erzielen, - die in ihrer äusseren Form einer willkürlichen Bewegung gleicht (vergl. S. 355). Demnach wäre die Art des Einzelreizes (ob linear oder momentan ansteigend) unwesentlich für die Geschwindigkeit der Zusammenziehung, und diese würde lediglich bedingt sein durch die Abstufung der Reizintensität und - was ich nicht untersucht habe - von der Reizfolge.

Nach der in der Arbeit vertretenen Auffassung, dass jede weitere Zunahme der Verkürzung von einer Heranziehung neuer Fasern abhängt, muss es eben belanglos sein, auf welche Weise gleichzeitig eine gewisse Anzahl von Fasern erregt wird. Das Wesentliche

1) F. Gotch, The effect of local injury upon the excitatory electrical response of nerve. Journ. of physiol. vol. 28 p. 32.1902. 
ist nur, dass von Impuls zu Impuls sich mehr Fasern an der Kontraktion beteiligen.

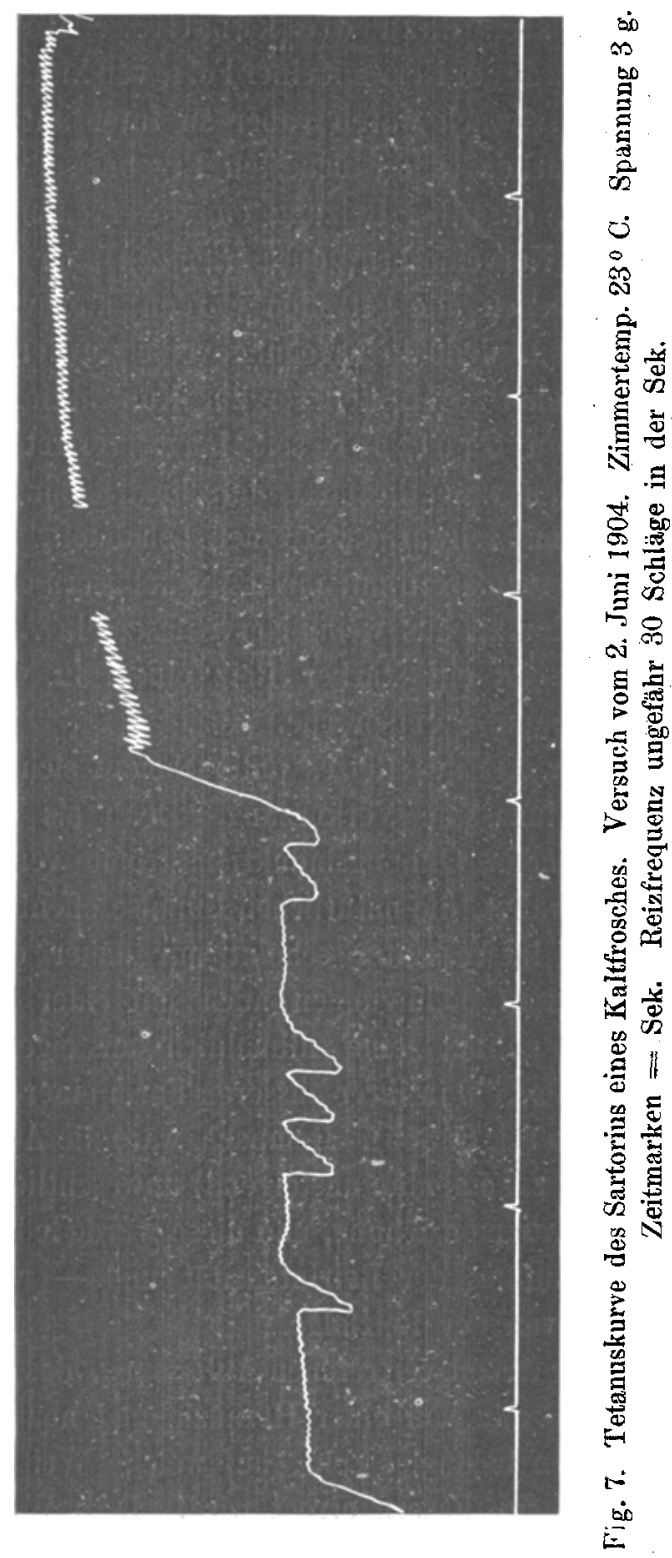

Bei dieser Gelegenlieit sei jedoch ausdrücklich darauf hingewiesen, dass nach der Grützner' schen Auffassung durchaus nicht bestritten 
Üb. den Einfl. der Reizstärke auf die Tètanuskurve des Froschsartorius. 361

werden soll, dass auch ein tind diesêbè Faser je nách der Reizung sich stark oder schwach zusammenziehen kann.

Die übrigen Eigenschaften der willkürlichen Muskelbewegung, nämlich das langsame Ansteigen der Aktionsströme und die geringe Frequenz der Oszillationen, müssen selbstverständlich auf die abweichende Art der natürlichen Reize zurückgeführt werden. Das Fehlen des sekundären Tetanus kann bedingt sein durch den langsamen Anstieg der natürlichen Reize oder durch den Umstand, dass in der Natur eben nie alle Fasern eines Muskels zusammen erregt werden, oder durch Beides.

\section{Versuche mit Kaltfröschen.}

Da der submaximale Tetanus des Froschgastrocnemius sehr häufig grosse Unregelmässigkeiten aufweist, spricht $\mathrm{Ho}$ ofman $n^{1}$ ) die Vermutung aus, dass dieselben durch Temperatureinfiüsse bedingt sind und möglicherweise speziell bei Kaltfröschen vorkommen. (Vgl. Fig. 7.)

Deshalb wollte ich feststellen, ob sich auch bei meinen Versuchen durch Abkühlung ein Unterschied im Tetanusverlauf hervorrufen lässt. Ich stellte einen Frosch vor dem Versuche einige Stunden in den Eisschrank und präparierte auch den Muskel auf einem mit Eis unterlegten Teller. Zum Anfeuchten benutzte ich stark abgekühlte Kochsalzlösung.

Bei dieser Versuchsanordnung trat ein submaximaler Tetanus auf, welcher häufige Unterbrechungen zeigte, an den zwischen diesen liegenden Stellen jedoch das gewöhnliche Aussehen hatte. (Vgl. Fig. 7.)

Während ich aber bei Warmfröschen die Kurve durch vorsichtiges Annähern des Schlittens beliebig langsam ansteigen lassen konnte, gelang dies bei Kaltfröschen nur viel schwieriger und weniger vollkommen, was allerdings aus obiger Kurve nicht zu ersehen ist. Die Kälte scheint also die physiologische Tätigkeit der beiden Faserarten im Muskel besser zu trennen, indem bei Verstärkung des Reizes plötzlich alle dicken Fasern mitgerissen werden.

Auf diese Versuche möchte ich indessen keinen zu grossen Wert legen, denn in dem während des verflossenen heissen Sommers ziemlich warmen Raume, in dem ich arbeitete, nahm der Muskel sicher bald die Temperatur der Umgebung an, weshalb ich auch diese

1) F. B. Hofmann, Über den Einfluss der Reizstärke auf den Tetanusverlauf bei indirekter Reizung. Dieses Arch. Bd. 95 S. 484 (492). 1903. 
Untersuchungen nicht weiter fortsetzte, da man Genaueres doch erst von speciell von diesem Gesichtspunkt aus vorgenommenen Untersuchungen erwarten darf.

\section{Gründe für die Verwendung des Sartorins.}

Den Sartorius verwendete ich zu meinen Versuchen, weil man wegen seines gleichmässigen parallelfaserigen Baues ein übersichtlicheres Ergebnis erwarten durfte als beim Gastrocnemius. Aber trotzdem wurden auch mit dem Wadenmuskel Kontrollversuche angestellt. Dabei gelang es zwar, einen submaximalen Tetanus zu erzielen, der durch Verstärkung des Reizes in den maximalen überging, aber nie batte ich Gelegenheit, bei einer Reizfrequenz, bei welcher der submaximale Tetanus glatt war, bei maximaler Verkürzung klonische Erzitterungen zu beobachten.

Dieses verschiedene Verhalten hat wohl teilweise seinen Grund in der grossen Masse des Gastrocnemius gegenüber dem Sartorius. Soweit sich der Unterschied darauf zurückführen lässt, wäre er also rein physikalischer Natur, indem eine grosse Masse schnellen Anstössen viel weniger folgen kann als eine kleine. Die grösste Schuld an der Verschiedenheit trägt aber vielleicht eine Tatsache, auf die ich erst im Laufe meiner Untersuchungen aufmerksam wurde. Sobald nämlich der Gastrocnemius längere Zeit tetanisiert war, erreichte er nach Aufhören des Reizes nicht sofort wieder seine frühere Länge, sondern verharrte in einer gewissen Verkürzung. Ganz anders verhielt sich der Sartorius: Auch wenn er noch so viele Tetanuskurven gezeichnet hatte, erreichte der Schreibhebel nach der Reizung in möglichst kurzer Zeit die Nulllinie.

Ein ähnlicher Unterschied dieser beiden Muskeln ist bei länger fortgesetzten Einzelzuckungen $\mathrm{zu}$ beobachten, indem der Gastrocnemius viel eher die für Ermüdung von Kaltblütermuske]n charakteristische Dehnung der Kurve aufweist als der Sartorius, worüber ich demnächst eingehender berichten werde.

Woher nun diese Erscheinung rührt, ob sie mit dem komplizierten Bau des Gastrocnemius zusammenhängt oder eine spezifische Eigenschaft seiner Muskelfasern bildet, darüber kann ich vorläufig noch nichts Bestimmtes sagen. Jedenfalls darf man aber diese Eigentümlichkeit als eine der Hauptursachen ansehen für das verschiedene Verhalten der beiden Muskeln im Tetanus. 
Üb. den Einfl. der Reizstärke auf die Tetanuskurve des Froschsartorius. 363

Der Grund, weshalb ich immer vom Nerven aus reizte, ist folgender.

Wie leicht einzusehen, wird bei der indirekten Reizung die unvermeidliche Polarisation in den Nerven verlegt und sich im Muskel höchstens durch Beeinflussung der Reizintensität fühlbar machen. Sicher aber arbeitet der Muskel unter normaleren Bedingungen, wenn er nicht durch polarisatorische Einflüsse geschädigt ist. Des weiteren wechselt bei direkter Reizung die Dichte und damit die Wirksamkeit des Stroms je nach dem Querschnitt des Muskels.

Es ist ausserdem ein grosser Unterschied, ob die Muskelfaser, wie es in der Natur stets gesehieht, vom Nerven aus den Impuls erhält, sich zu kontrahieren, oder ob die Erregung immer an einer und derselben Stelle erfolgt, die allmählich ermüden muss, da ja die Reizung immer unter der Kathode erfolgt. Dass es nicht gleichgültig ist, ob ein Muskel direkt oder von seinem Nerven aus gereizt wird, geht schon aus der Tatsache hervor, dass bei direkter Durchleitung des Stromes die tonischen Erscheinungen viel mehr in den Vordergrund treten als bei Reizung des Nerven, - eine Tatsache, die meines Wissens zuerst von $\mathrm{Ti}_{\mathrm{ege}} \mathbf{l}^{\mathbf{1}}$ ) hervorgehoben und genauer untersucht wurde.

\section{Zusammenfassnung der Ergebnisse.}

1. Der Sartorius von Rana temp. lässt sich bei faradischer Reizung von seinem Nerven aus leicht in einen submaximalen und durch Verstärkung des Reizes in einen maximalen Tetanus versetzen.

2. Der submaximale Tetanus wird in der Regel glatt bei einer Reizfrequenz von 25-27 Schlägen in der Sekunde.

3. Der maximale Tetanus wird erst glatt bei einer Reizfrequenz von ungefähr 34 .

4. Wenn man also den Sartorius reizt mit einer Schlagfolge, die zwischen 27 und 34 liegt, erhält man bei schwachen Reizen einen niederen glatten, bei starken einen bohen gezackten Tetanus.

5. Dieses Verhalten des Sartorius ist dadurch zu erklären, dass beim Tetanisieren mit schwachen Strömen die leicht erregbaren,

1) E. Tieg el, Über Muskelkontraktur im Gegensatz zu Kontraktion. Dieses Arch. Bd. 13 S. 71 (73). 1876. 
364. Adolf Basler: Üb. den Einfl. der Reizstärke auf die Tetanuskurve etc.

langsamen, dünnen Fasern erregt werden, bei starken Reizen dagegen die schwerer erregbaren, an Kraft überlegenen, flinken, dicken Fasern.

6. Mit dem vom Nerven aus gereizten Sartorius gelingt es auch, dem Tetanus den Verlauf einer natürlichen Bewegung zu geben, d. h. den Muskel sich beliebig langsam kontrahieren zu lassen.

7. Zur Ausführung der beschriebenen Versuche scheint sich der Gastrocnemius nicht zu eignen.

8. Die Gültigkeit vorstehender Tatsachen ist zunächst bewiesen für Sommerfrösche und für eine Zimmertemperatur zwischen 22 und $25^{\circ} \mathrm{C}$.

Für die Anregung zu dieser Arbeit und für die gütige Unterstützung bei ihrer Ausführung spreche ich Herrn Professor v. Grützner auch an dieser Stelle meinen verbindlichsten Dank aus. 